\title{
RELACIÓN ENTRE EL DIAGNOSTICO INTELECTUAL Y EL DESARROLLO SOCIAL EN RETARDADOS MENTALES LEVES Y FRONTERIZOS
}

\author{
Amparo Sótil de Pacheco
}

\begin{abstract}
RESUMEN
El presente estudio es fruto de la experiencia profesional durante años, en el tratamiento de niños y adolescentes con retardo mental educable que cursan estudios en el Centro de Educación Especial $N^{\circ} 06$.

Pretende ser una contribución al conocimiento cabal de la problemática del educando con retardo mental, en lo referente a la relación inteligencia y adaptación social.

El Sistema Educativo a través de la modalidad de Educación Especial, viene implementando la apertura de Talleres donde se imparte capacitación laboral a los educandos, a fin de calificarlos en el desempeño de una actividad laboral especifica que es lo que posibilitara la rehabilitación del excepcional mediante su integración económica a la sociedad, permitiéndoles encontrar una vida con sentido y digna de un ser humano.

Teniendo en cuenta la complejidad para seleccionar, ubicar y adiestrar en un taller compatible con sus aptitudes, intereses y potencialidades, es necesario contar con métodos $v$ procedimientos válidos y confiables, que permitan una aproximación científica al conocimiento y solución de los problemas planteados.
\end{abstract}

Palabras clave: Retardo Mental, Adaptación Social, Diagnostico Intelectual, R.M Leve, Categoría Mental, Fronterizo.

\begin{abstract}
The present study is fruit of the professional experience during years, in the treatment of children and teenagers with educable mental retardation that study in the Center of Special Education $N^{\circ} 06$.

It seeks to be a contribution to the exact knowledge of the problem of the student with mental retardation, regarding the relationship between intelligence and social adaptation.

The Educational System through the modality of Special Education, comes implementing the opening of Shops where labor training is imparted to the students, in order to qualify them in the acting of a labor activity it specifies that it is what facilitated the rehabilitation of the exceptional one by means of their economic integration to the society, allowing them to find a life with sense and worthy of a human being.

Keeping in mind the complexity to select, to locate and to train in a compatible shop with their aptitudes, interests and potentialities, it is necessary to have methods and valid and reliable procedures that allow a scientific approach to the knowledge and solution of the outlined problems.
\end{abstract}

Key Words: I slow Mental, Social Adaptation, Intellectual Diagnostic, R.M. Light, Borderline. 


\section{ESTABLECIMIENTO DEL PROBLEMA}

El propósito primordial de este estudio es determinar la relación existente entre inteligencia y adaptación social en el escolar con Retardo Mental Educable, mediante la Escala de Inteligencia de Wechsler y la prueba de Desarrollo Social para Retardados Mentales Leves, P.A.C., Forma 2, HeC. Gunzburg.

Se incide especialmente en hacer un diagnóstico intelectual del potencial de habilidades y destrezas, así como también el pronóstico de desarrollo social, incidiendo en las áreas de independencia personal, comunicación socialización y ocupación. El objetivo fue determinar las áreas de menor rendimiento en las cuales se brindaría mayor apoyo. El diagnóstico intelectual y los puntajes de los niveles de desarrollo social nos puede dar pautas para su ubicación en el taller y para el plan pedagógico terapéutico, que puede ser apoyado por el psicólogo, profesor y padres de familia.

\section{FORMULACIÓN DE LA HIPÓTESIS}

Se presume que:

No existirán concordancias entre los diagnósticos intelectuales de Educables y Fronterizos y los logros alcanzados en las áreas de independencia personal, comunicación socialización y ocupación, evaluados a través del Wechsler y del P.A.C.

\section{INVESTIGACIONES PREVIAS}

Trabajos nacionales sobre este aspecto en nuestro medio son pocos. Se han realizados muchas investigaciones sobre el área de retardo mental. pero desde otras perspectivas: relación entre el ajuste social" las habilidades sociales y el éxito profesional en el retrasado mental, merece citarse el elaborado por Ruiz Alva (1989) quien ha utilizado la misma prueba empleada en este trabajo (P.A.C.). Sostiene que en la muestra estudiada los rendimientos alcanzados son bajos en las áreas de comunicación, socialización y ocupación, por lo que se infiere que aún necesitan entrenamiento en el aspecto social y no están preparados para el ajuste armónico con su medio.

Otros autores extranjeros han trabajado en esta área, Cowan y Goldman (1989) opinan que el nivel de la educación y las experiencias laborables previas no son factores significativos para el ajuste profesional.

Engle (1982) realizó un estudio muy extenso sobre el empleo del adulto retrasado mental. Menciona recomendaciones que parecen importantes para el éxito:

1. El ajuste profesional y social debe recibir una atención especial.

2. Los problemas escolares deberán centrarse en torno del ajuste personal, de las actitudes sociales y de ciertos hábitos laborales idóneos.

3. Debemos brindar un consejo de tipo personal y profesional al retrasado mental antes que termine el período de escolaridad.

\section{MÉTODO}

\section{Muestra}

Fueron seleccionados 33 sujetos (varones y mujeres) que asistían a las secciones de Talleres Ocupacionales, que vienen adiestrándose en las áreas ocupacionales que se imparten en el Centro de educación Especial $N^{\circ} 06$, de las cuales 25 presentaban un 
diagnóstico de Retardo Mental Educable y 8 de Fronterizos. A esta institución acuden educandos de nivel socio económico bajo.

\section{Instrumentos}

a) Ficha de Evaluación del Desarrollo Social para Retar dados Mentales Leves, P.A.C., Forma 2.

Esta prueba fue elaborada por Gunzburg, Director de los Servicios Psicológicos del Hospital de Moyhull - Inglaterra.

Explora cuatro áreas:

1. Independencia Personal: En este rubro se analizan los hábitos y habilidades que tienen que ver con las conductas de auto-valimiento, referidas a: comportamiento en la mesa, posibilidades de desplazamiento, la higiene y el vestirse.

2. Comunicación: Esta área abarca el estudio del nivel de comprensión y expresión del lenguaje verbal y simbólico, es decir: lectura, escritura y números.

3. Socialización: Aquí se detallan las habilidades y conductas referidas a compras, manera de comportarse, ayuda en el hogar, manejo de dinero.

4. Ocupación: Esta área comprende destrezas y habilidades necesarias para la realización de una actividad laboral, mide aspectos como: actividades manuales, ocupación del tiempo libre, calidad y esmero en el trabajo, así como el cuidado de herramientas y material de trabajo.

\section{Técnica de Administración}

Se administra a la madre o familiar que tenga a su cargo al probando mediante una entrevista; explicándole previamente la necesidad de ser sincera al responder, pues de ello depende que se elabore un adecuado programa conectivo para el alumno.

El cuestionario consta de 120 ítemes de los resultados se traducirán en un gráfico y en términos porcentuales, señalando asimismo las conductas que necesitan entrenamiento y las sugerencias dadas por el examinador.

\section{VALIDEZ}

La prueba del P.A.C., ha demostrado ser un instrumento suficientemente válido para trabajos experimentales y de Diagnóstico. Gunzburg señala que los datos de la validez concurrente, de construcción y predictiva, son adecuados, seguidos del análisis del contenido y del diagnóstico.

Los resultados obtenidos correlacionan positivamente con las evaluaciones académicas de los profesores, principalmente con el área de comunicación.

\section{b) La Escala de inteligencia de Wechsler}

Fue seleccionada en base a que explora habilidades verbales y ejecutivas, además de dar un diagnóstico Intelectua1.

\section{PRESENTACIÓN Y DISCUSIÓN DE LOS RESULTADOS}

En las Gráficas 1 y 2, aparecen frecuencias alcanzadas en los diversos niveles de Desarrollo Social evaluadas con el PA.C. así como también las logradas en las diversas áreas de la prueba: Independencia Personal, Comunicación, Socialización y Ocupación. 
Gráfice No, 1

Niveles de Desarrollo Social de Fronterizos

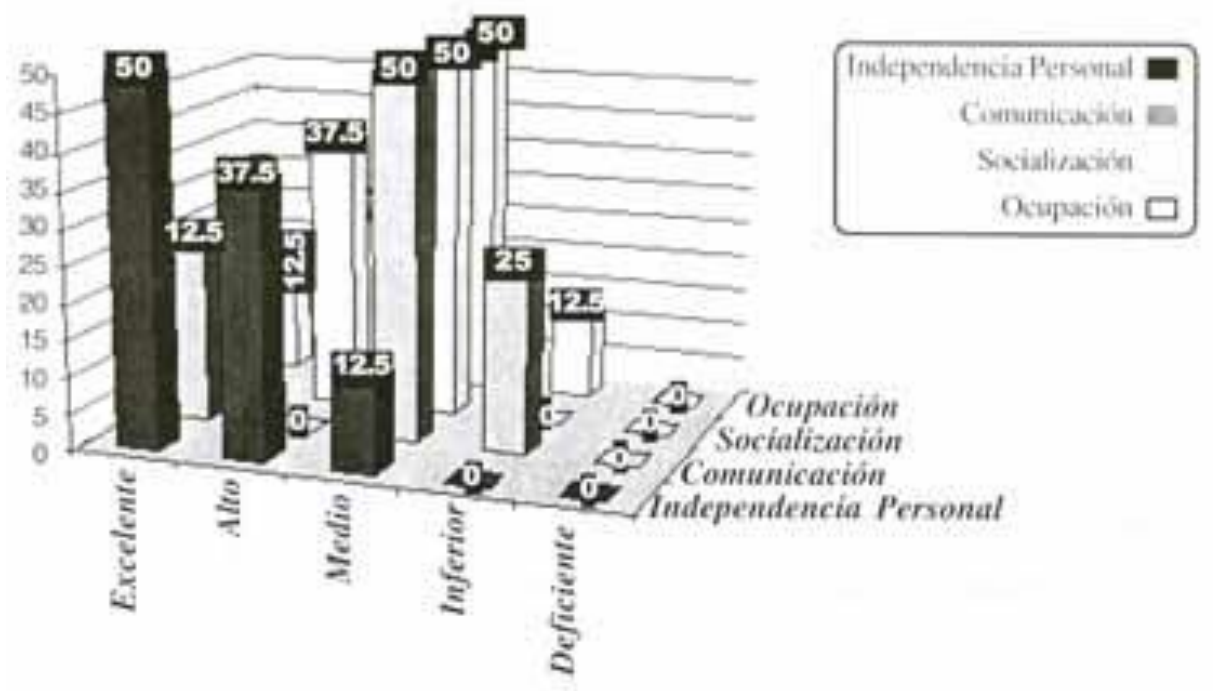

Pudiéndose observar que el mayor porcentaje se ubica en el nivel de diagnóstico Social de Excelente, tanto en el grupo de Retardo Mental Leve (52\%), como en el de Fronterizos (50\%); y que en las diferentes áreas los mayores porcentajes se concentran en los niveles de diagnóstico Medio del P.A.C., tanto para Educables como para Fronterizos.

Grafice No, 2

Niveles de desarrollo social de sujetos educables

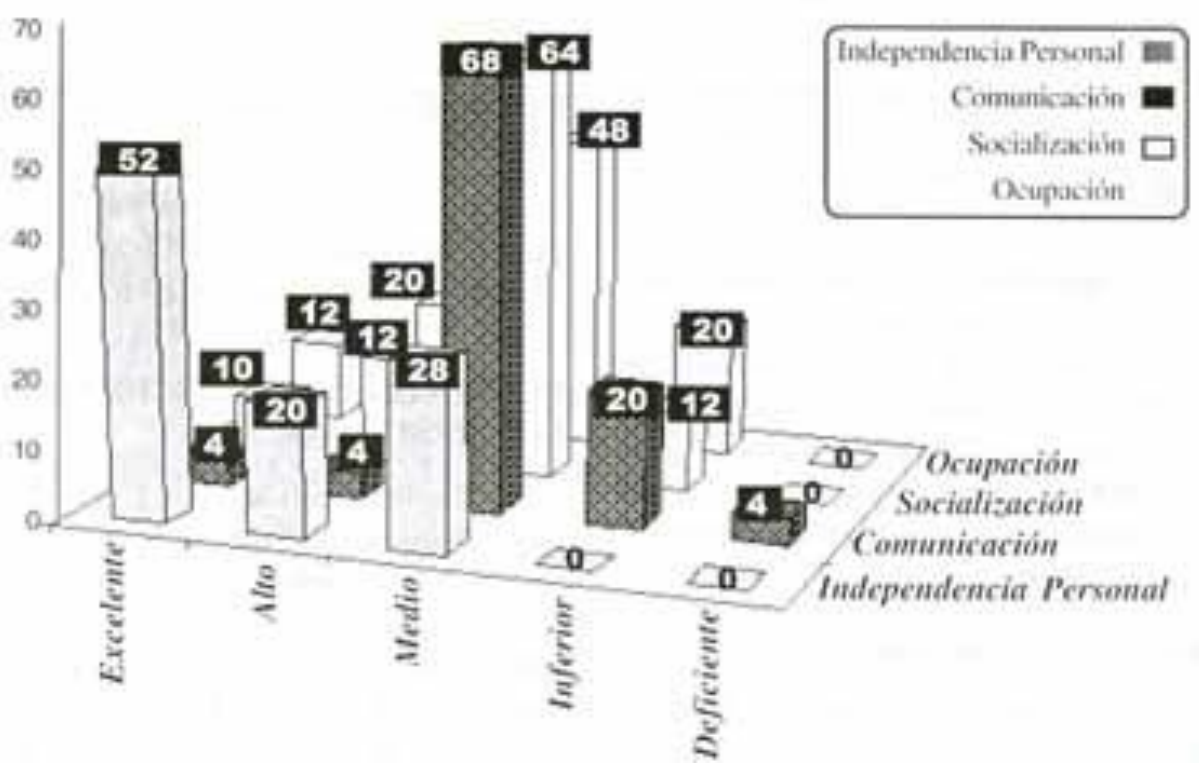


Los hallazgos encontrados en relación a la asociación e independencia entre el diagnóstico Intelectual y el Desarrollo Social en sujetos con retardo mental educables y fronterizos, demuestran que, no existen asociaciones estadísticamente significativa entre dichas variables tal como se puede observar en la Tabla $\mathrm{N}^{\circ} 1$.

Tabla $\mathbf{N}^{\circ} 1$

Valores de $x^{2}$ aplicados a la hipótesis de que los niveles de desarrollo social son independientes del diagnostico intelectual

\begin{tabular}{|c|c|c|c|c|c|c|}
\hline \multirow{2}{*}{$\begin{array}{c}\text { Intelectual } \\
\text { Intico }\end{array}$} & \multicolumn{2}{|c|}{ N I V E L E S } & D E & \multicolumn{2}{c|}{ D I A G N O S T I C O } & \multirow{2}{*}{ TOTAL } \\
\cline { 2 - 7 } & Excelente & Alto & Medio & Inferior & Deficiente & \\
\hline Educables & 0.032 & 0.528 & 0.182 & 0 & 0 & 0.724 \\
Fronterizos & 0.099 & 1.658 & 0.569 & 0 & 0 & 2.236 \\
\hline TOTAL & $\mathbf{0 . 1 3 1}$ & $\mathbf{2 . 1 8 6}$ & $\mathbf{0 . 7 5 1}$ & $\mathbf{0}$ & $\mathbf{0}$ & $\begin{array}{c}\mathbf{3 . 0 6 8} \\
\text { NS }\end{array}$ \\
\hline
\end{tabular}

NS. No Significativo

G.L. : 4

$X^{2} 0.05 \quad 9.488$

Estos resultados traducen la efectividad del sistema escolarizado a través de la estructura curricular, ejerciendo un rol socializante del educando, que posibilita el desempeño de conductas adecuadas al margen del potencial intelectual que presentan los grupos materia de estudio, corroborándose de esta forma la aseveración de Robert Isaacson, quien expresa que el Cociente Intelectual no es un elemento de juicio adecuado para determinar si un individuo podrá manejarse con éxito en la sociedad; es probable que ello dependa de otras características mentales y de conducta que distinguen a los individuos.

Por otro lado, se ha efectuado un análisis de los niveles de logros alcanzados en las diferentes áreas del P.A.C. y los diagnósticos intelectuales, pudiéndose concluir que entre el área de Independencia Personal y la inteligencia tampoco existe asociación, tal como aparece en la Tabla $\mathrm{N}^{\circ}$ 2. Esto revela que, tanto los Educables como Fronterizos poseen conductas de auto-valimiento, como: hábitos en la mesa, posibilidades de desplazamiento, cuidado de la ropa, higiene y vestirse; las mismas que han sido reforzadas y ampliadas en la etapa de aprestamiento y conocimientos básicos, que anteceden a la etapa de Capacitación Laboral y que tienen un proceso longitudinal en la acción educativa en donde se supone que este bagaje de experiencias constituye el perfil básico del programa educativo.

Los contenidos evaluados en este rubro, son los que se vienen trabajando en el hogar y la escuela. 


\section{Tabla $\mathbf{N}^{\circ} 2$}

Valores de $x^{2}$ aplicados a la hipótesis de que los niveles en el área de independencia personal son independientes del diagnostico intelectual

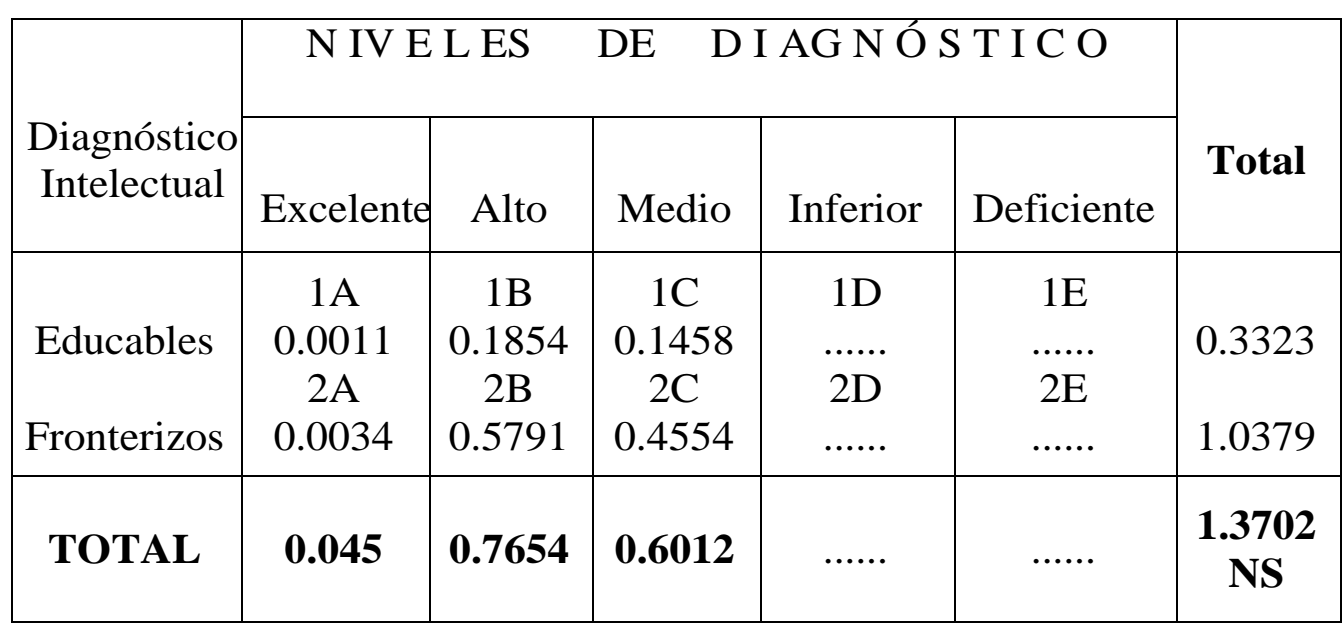

N.S. No significativo

G.L. : 4

$x^{2} 0.05$

Tabla $N^{\circ} 3$

Valores de $x^{2}$ aplicados a la hipótesis de que los niveles en el área de comunicación son independientes del diagnóstico intelectual

\begin{tabular}{|c|c|c|c|c|c|c|}
\hline \multirow{2}{*}{$\begin{array}{c}\text { Diagnóstico } \\
\text { Intelectual }\end{array}$} & \multicolumn{2}{|c|}{ N IV E L ES } & \multicolumn{3}{|c|}{ DE D I AG N Ó S T I C O } & \multirow[b]{2}{*}{ Total } \\
\hline & Excelente & Alto & Medio & Inferior & Deficiente & \\
\hline \multirow{3}{*}{ Educables } & $1 \mathrm{~A}$ & $1 \mathrm{~B}$ & $1 \mathrm{C}$ & 1D & $1 \mathrm{E}$ & \\
\hline & 0.71 & 0.08 & 0.08 & 0.02 & 008 & 0.97 \\
\hline & $2 \mathrm{~A}$ & $2 \mathrm{~B}$ & $2 \mathrm{C}$ & $2 \mathrm{D}$ & $2 \mathrm{E}$ & \\
\hline Fronterizos & 2.21 & 0.24 & 0.23 & 0.05 & 0.24 & 2.97 \\
\hline TOTAL & 2.92 & 0.32 & 0.31 & 0.07 & 0.32 & 3.94 \\
\hline
\end{tabular}

N.S. No significativo

G.L. : 4

$x^{2} 0.05 \quad 9.488$

Como podemos observar en las Tablas No. 3. 4 Y 5. la relación entre el Diagnóstico Intelectual y los niveles alcanzados por el PA.C. tampoco arrojan valores significativos al ji cuadrado. 
Tabla $\mathrm{N}^{\circ} 4$

Valores de $x^{2}$ aplicados a la hipótesis de que los niveles en el área de socialización son independientes del diagnostico intelectual

\begin{tabular}{|c|c|c|c|c|c|c|}
\hline \multirow{2}{*}{$\begin{array}{l}\text { Diagnóstico } \\
\text { Intelectual }\end{array}$} & \multicolumn{5}{|c|}{ N IV E L ES DE D I AG N Ó S T I C O } & \multirow{2}{*}{ Total } \\
\hline & Excelente & Alto & Medio & Inferior & Deficiente & \\
\hline $\begin{array}{l}\text { Educables } \\
\text { Fronterizos }\end{array}$ & $\begin{array}{c}1 \mathrm{~A} \\
0.03 \\
2 \mathrm{~A} \\
0.10\end{array}$ & $\begin{array}{c}1 \mathrm{~B} \\
0.32 \\
2 \mathrm{~B} \\
1.00\end{array}$ & $\begin{array}{c}1 \mathrm{C} \\
0.05 \\
2 \mathrm{C} \\
0.15\end{array}$ & $\begin{array}{c}1 \mathrm{D} \\
0.23 \\
2 \mathrm{D} \\
0.73\end{array}$ & $\begin{array}{l}1 \mathrm{E} \\
\ldots . . \\
2 \mathrm{E} \\
\ldots . .\end{array}$ & 0.63 \\
\hline TOTAL & 2.92 & 0.32 & 0.31 & 0.07 & 0.32 & 3.94 \\
\hline
\end{tabular}

N.S. No significativo

G.L. : 4

$\begin{array}{lll}x^{2} & 0.05 \quad 9.488\end{array}$

En comunicación, los niveles de rendimiento son medios, tanto para educables como fronterizos lo que permite la adecuada comunicación en sus aspectos: comprensivo, expresivo y simbólico.

Tabla $N^{\circ} 5$

Valores de $x^{2}$ aplicados a la hipótesis de que los niveles en el área de ocupación son independientes del diagnóstico intelectual

\begin{tabular}{|l|c|c|c|c|c|c|}
\hline \multirow{2}{*}{$\begin{array}{l}\text { Diagnóstico } \\
\text { Intelectual }\end{array}$} & \multicolumn{3}{|c|}{ N IV E L ES DE D I AG N Ó S T I C O } & \multirow{2}{*}{ Total } \\
\cline { 2 - 6 } & Excelente & Alto & Medio & Inferior & Deficiente & \\
\hline \multirow{3}{*}{ Educables } & $1 \mathrm{~A}$ & $1 \mathrm{~B}$ & $1 \mathrm{C}$ & $1 \mathrm{D}$ & $1 \mathrm{E}$ & \multirow{2}{*}{0.025} \\
& 0.003 & 0.017 & 0.001 & 0.004 & $\ldots .$. & \\
Fronterizos & $2 \mathrm{~A}$ & $2 \mathrm{~B}$ & $2 \mathrm{C}$ & $2 \mathrm{D}$ & $2 \mathrm{E}$ & \\
& 0.009 & 0.052 & 0.003 & 0.139 & $\ldots .$. & \multirow{2}{*}{0.203} \\
\hline TOTAL & $\mathbf{0 . 0 1 2}$ & $\mathbf{0 . 0 6 9}$ & $\mathbf{0 . 0 0 4}$ & $\mathbf{0 . 1 4 3}$ & $\ldots .$. & $\mathbf{0 . 2 2 8}$ \\
\hline
\end{tabular}

N.S. No significativo

G.L. : 4

$x^{2} 0.05 \quad 9.488$ 


\section{CONCLUSIONES}

$\mathrm{Al}$ analizarse las relaciones entre los diagnósticos de Educables y Fronterizos y los niveles alcanzados en Desarrollo Social y las diversas áreas evaluadas por el P.A.C., se ha podido arribar a las siguientes conclusiones:

1. Los mayores porcentajes en relación al desarrollo social se encuentran en un Nivel Medio, tanto para Educables como Fronterizos.

2. Los mayores porcentajes en las áreas de Comunicación Socialización y Ocupación para ambos grupos, se hallan en el Nivel de diagnóstico Medio.

3. En Independencia Personal el porcentaje es más elevado, $52 \%$ Excelente para Educables y $50 \%$ para Fronterizos.

4. No existe relación entre las categorías di agnósticas de Inteligencia y los Niveles de Desarrollo Social, lo que expresaría que el nivel mental no es tan importante como los rasgos personales y las características del trabajador.

5. Así mismo, no existe relación entre los niveles diagnósticos de Educables y Fronterizos y las di versas áreas de Independencia Personal, Comunicación, y ocupación, lo que revela que estas habilidades son debidamente entrenadas tanto en la escuela como en el hogar.

\section{BIBLIOGRAFIA}

Aguilera, M. J. y otros. Evaluación del Programa de integración de alumnos con deficiencias. CIDE - Madrid. 1990.

Araujo, C. y Diz, A. Integración Laboral de disminuidos psíquicos. 1992.

Benedet, María Jesús. Procesos cognitivos en la deficiencia mental. 1991.

Brennan, W.K. El currículo para niños con necesidades educativas especiales. Siglo XXI-MEC-Madrid. 1998.

Briones, G. La edición Retardo Mental. Conceptos para un cambio Institucional. Ed. Trillas. México. 1986.

Diaz Alcaraz, M. Formación Profesional y Empleo del Deficiente Psíquico. Ed. Gráficas Torova Fuenlabrada. Madrid. 1980.

García Pérez, Carmen. Integración Socio Laboral del Deficiente Mental Psíquico. 1995.

Hutk, Maxl. Los niños con Retardo Mental. Desarrollo Aprendizaje y Educación. 1988.

Ibañez, P. El mundo laboral del deficiente mental. UNED. Madrid. 1988.

Muntaner, Joao. La Sociedad ante el Deficiente Mental. Narcea S.A. Madrid. 1995. 